\title{
Onset of Buoyancy and Surface-Tension Driven Instabilities in Presence of Random Vibrations
}

\author{
B. S. Dandapat \\ Physical and Earth Science Division, Indian Statistical Institute, Calcutta, India
}

Z. Naturforsch. 45a, 1235-1240 (1990); received August 10, 1990

\begin{abstract}
Onset of thermal convection in an incompressible fluid layer bounded between a perfectly heat conducting lower rigid plate and an upper free surface is analysed when the layer is subject to random vibrations. It is shown that when the vibrations are characterized by a white noise process, they hasten the onset of convection. Further it is shown that the stability zone is demarcated by an inverted parabola in the $(R, M)$ plane.
\end{abstract}

\section{Introduction}

The experiments of Bénard [1] marked the beginning of the study of fluid motion in a shallow layer of fluid heated uniformly from below. Rayleigh's [2] theoretical analysis for the onset of thermal convection seemed to provide a basis for the understanding of the Bénard cell. In this analysis, Rayleigh showed that a critical temperature gradient is necessary for the onset of convection. In his later experiments [3], Bénard had observed convection at much smaller temperature gradients. Block [4] resolved this discrepancy by showing in his experiments the significant role played by surface tension. Pearson [5] gave a proper mathematical account for the surface-tension driven instability. He showed that the variation of surface tension with temperature could lead to convection in a heated fluid layer, even in the absence of buoyancy. This effect is due to the shearing force produced in the surface layer of a fluid by surface tension gradients and has been known in the literature as the Marangoni effect. Afterwards, Nield [6] studied the combined effect of surface tension and buoyancy on the onset of thermal convection. Scriven and Sternling [7] neglected gravity but considered the effect of fluid depth. He found that the onset of convection might occur at very small temperature differences.

Davis [8] studied the buoyancy and surface-tension driven instability and found a subcritical instability in a small range of Marangoni numbers for sufficiently small Rayleigh numbers. Subsequently Davis and Homsy [9] and Castillo and Velarde [10] also con-

Reprint requests to Dr. B. S. Dandapat, Physical and Earth Science Division, Indian Statistical Institute, 203 B.T. Road, Calcutta-700035, Indien. cluded that a subcritical instability exists. Nonlinear theoretical studies of surface-tension driven convection have also been made by Scanlon and Segel [11], Kraska and Sani [12], Rosenblat, Davis and Homsy [13] and Cloot and Lebon [14].

Some literature exists on the effect of random noise on the onset of thermal convection with a specified time-dependent heat input. Ahlers, Cross, Hohenberg and Safran [15] discussed the state of flow near the onset of Bénard convection on the hypothesis that, if the system remains close to the threshold of convection, its resonance can be described by an amplitude equation derived by Segel [16] and Newell and Whitehead [17] with a suitable forcing field to account for the random forcing. In particular, Ahlers et al. [15] showed that when the random forcing is a Gaussian variable with zero mean and a white-noise spectrum, their amplitude equation can satisfactorily describe the results of experiments with a heat input in the form of a linear ramp. Further, on analyzing their experimental data for long times, they found that the state first excited in the presence of random forcing has not the roll pattern obtained in the steady state but a hexagonal pattern. This study clearly reveals the importance of stochastic effect on the pattern formation in the initial stages of convection. This result was further experimentally confirmed by Mayer, Ahlers and Cannell [18] in their flow visualization and heat flux studies during the initial stages of the evolution of Bénard convection in a cylindrical container with aspect ratio (radius/height) 10 . The random forcing in the above experiment was in the form of a heat current which was ramped linearly or modulated periodically with respect to time. By using a novel design, they eliminated the influence of the side walls on the pattern 
formation. It was found that in the initial stages the pattern consisted of irregularly arranged cells similar to the hexagonal pattern observed in [15]. It may be mentioned that prior to the above studies, Zaitsev and Shliomis [19] investigated Bénard convection with a random forcing based on a linear theory. They found that the critical Rayleigh number $R_{\mathrm{c}}$ of linear stability theory remains unaffected by random forcing but the forcing leads to an imperfect bifurcation near the eigenvalue of the linear theory. On the other hand, Jhaveri and Homsy [20] studied the Bénard convection near the threshold based on a theory of weakly non-linear convection in the presence of random fluctuations of thermodynamic origin arising in the system itself. They found that, as in [15] and [18], random forcing is important in the initial stages and the evolution of ordered convection takes place through three stages of pattern formation.

In all the above studies, the influence of surface tension on the onset of convection was neglected. In this paper we study the onset of convection subject to random forcing in a horizontal layer of fluid with a free surface, taking into account the influence of surface tension. In particular we assume the random forcing in the form of white noise. This work may help in understanding crystal growth in a low-orbiting spacecraft which experiences random vibrations in the frequency range roughly from 0.1 to $10 \mathrm{~Hz}$ (Ostrach [21]). Apart from its practical significance, the problem has some methodological interest since the stability analysis is reduced to the study of a certain class of stochastic (or random) differential equations. We shall, however, confine ourselves to the mean squares stability of the solution of such equations.

\section{Mathematical Formulation and Stability Analysis}

Consider the basic state of a horizontal incompressible fluid layer of thickness $d$ between a perfectly heat conducting lower rigid plate and an upper free surface. The layer is infinite in the horizontal $x$ and $y$ directions and is heated from below.

The temperatures $T_{1}$ at $z=0$ and $T_{2}$ at $z=d$ are constant. We define $\beta=\left(T_{2}-T_{1}\right) / d$. Further we assume that the total vertical acceleration is $g+g^{\prime}(t)$, where $g$ is the gravitational acceleration and $g^{\prime}(t)$ is a stochastic acceleration caused by vibration of the lower plate.

Let $\theta$ be the temperature deviation from the basic state and $(u, v, w)$ the components of the velocity field $q$ in the perturbed state. Using the Boussinesq approximation and eliminating all the variables in favour of the dimensionless vertical velocity $w_{1}(=w d / v$, where $v$ is the kinematic viscosity) and the dimensionless temperature perturbation $\theta_{1}(\theta / \beta d)$, we obtain the dimensionless equations

$$
\begin{aligned}
& \frac{\partial}{\partial \tau} \nabla^{2} w_{1}=\left(g \alpha \beta d^{4} / v\right)[1+G(\tau)] \nabla_{1}^{2} \theta_{1}+\nabla^{4} w_{1}, \\
& \operatorname{Pr}\left[\frac{\partial \theta_{1}}{\partial \tau}+w_{1}\right]=\nabla^{2} \theta_{1},
\end{aligned}
$$

where use has been made of the scaling factors $d^{2} / v$ and $d$ for time and length, respectively. $\nabla_{1}^{2}$ and $\nabla^{2}$ are the two and three dimensional Laplacian operators. The Prandtl number $\operatorname{Pr}$ and $G(\tau)$ are defined as

$$
\operatorname{Pr}=v / k, \quad G(\tau)=g^{\prime}(t) / g,
$$

where $k$ is the heat diffusivity, $\alpha$ is the thermal expansion coefficient.

The boundary conditions are at $z=0$ :

$$
w_{1}=0, \quad \partial w_{1} / \partial z=0, \quad \theta_{1}=0,
$$

and at $z=1$ :

$$
\begin{aligned}
& w_{1}=0, \quad \partial^{2} w_{1} / \partial z^{2}=(M / P r) \nabla_{1}^{2} \theta_{1} \quad(M>0), \\
& \partial \theta_{1} / \partial z=-h \theta_{1} \quad(h>0),
\end{aligned}
$$

with $h$ the Biot heat transfer coefficient.

The relations (3) express that the lower surface is rigid and perfectly heat conducting while (4) dscribes a non-deformable surface with a temperature dependent surface tension $\sigma(T)$. The Marangoni number $M$ is defined by

$$
M=\left[|\partial \sigma / \partial T| \beta d^{2}\right] /(\mu k),
$$

where $\mu$ is the viscosity.

Following normal mode technique (Chandrasekhar [22]) we take

$$
\begin{aligned}
& w_{1}=W(z, \tau) \exp \left[i\left(k_{x} x+k_{y} y\right)\right], \\
& \theta_{1}=\Theta(z, \tau) \exp \left[i\left(k_{x} x+k_{y} y\right)\right],
\end{aligned}
$$

where $W(z, \tau)$ and $\Theta(z, \tau)$ are assumed in the form (Finlayson [23])

$$
\begin{aligned}
& W(z, \tau)=\sum A_{i}(\tau) w_{i}(z) \quad \text { and } \\
& \Theta(z, \tau)=\sum B_{i}(\tau) \Theta_{i}(z),
\end{aligned}
$$

where $A_{i}(\tau)$ and $B_{i}(\tau)$ are unknown time dependent amplitudes. Here both $w_{i}(z)$ and $\Theta_{i}(z)(i=1,2, \ldots)$ constitute a complete set of functions satisfying the 
boundary conditions

$$
w_{1}(0)=D w_{i}(0)=\Theta_{i}(0)=0 \text { and } w_{i}(1)=0,
$$

where $D$ stands for $\mathrm{d} / \mathrm{d} z$.

Following the method of weighted residuals due to Finlaysan [23], we substitute (5) and (6) in (1) and (2) and obtain, after elimination of $A_{i}$, the following equation for $B_{i}$ :

$\operatorname{Pr} C_{j i} F_{j i} \frac{\mathrm{d}^{2} B_{i}}{\mathrm{~d} \tau^{2}}+\left[C_{j i} G_{j i}+\operatorname{Pr} D_{j i} F_{j i}\right] \frac{\mathrm{d} B_{i}}{\mathrm{~d} \tau}$

$+\left[D_{j i} G_{j i}-M H_{j i} U_{j i}-R V_{j i} H_{j i}\right] B_{i}=R G(\tau) V_{j i} H_{j i} B_{i}$,

where

$$
\begin{aligned}
& C_{j i}=\left\langle D W_{j} D W_{i}+a^{2} W_{j} W_{i}\right\rangle, \\
& D_{j i}=\left\langle D^{2} W_{j} D^{2} W_{i}+2 a^{2} D W_{j} D W_{i}+a^{4} W_{j} W_{i}\right\rangle, \\
& U_{j i}=-a^{2} D W_{j}(1) \Theta_{j}(1), \\
& V_{j i}=\left\langle a^{2} W_{j} \Theta_{i}\right\rangle, \\
& H_{j i}=\left\langle\Theta_{j} \mathrm{~W}_{i}\right\rangle, \\
& F_{j i}=\left\langle\Theta_{j} \Theta_{i}\right\rangle, \\
& G_{j i}=\left\langle D \Theta_{j} D \Theta_{i}+a^{2} \Theta_{j} \Theta_{i}\right\rangle+h \Theta_{j}(1) \Theta_{i}(1), \\
& a^{2}=k_{x}^{2}+k_{y}^{2}
\end{aligned}
$$

and

$$
\langle\cdots\rangle=\int_{0}^{1}(\cdots) \mathrm{d} z .
$$

The Reighlay number $R$ is defined as

$$
R=\left(g \alpha \beta d^{4} / v k\right) \text {. }
$$

Finally (8) reduces to

$\ddot{B}_{i}+C_{1} \dot{B}_{i}+D_{1} B_{i}=\frac{R V_{j i} H_{j i}}{\operatorname{Pr} C_{j i} F_{j i}} G(\tau) B_{i}$,

where

$$
\begin{aligned}
& C_{1}=\left(D_{j i} / C_{j i}\right)+\left(G_{j i} / \operatorname{Pr} F_{j i}\right), \\
& D_{1}=\left(D_{j i} G_{j i}-M H_{j i} U_{j i}-R V_{j i} H_{j i}\right) /\left(\operatorname{Pr} C_{j i} F_{j i}\right) .
\end{aligned}
$$

Overdots denote differentiation with respect to $\tau$. It may be seen from (10) that in addtion to the timedependent stochastic parameter $G(\tau)$, the stability characteristics depend on the Rayleigh number $R$, the Prandtl number $\operatorname{Pr}$ and the Marangoni number $M$.

Instead of attempting to find the explicit solution of (10), we shall seek the mean squares stability criterion for this solution. This implies that every mean squares bounded input leads to a mean squares bounded output. To this end we assume that $G(\tau)$ is given by a white noise process and recast (10) as a two-dimensional Ito equation given by

$$
\mathrm{d} \boldsymbol{X}(\tau)=\boldsymbol{f}(\boldsymbol{X}(\tau), \tau) \mathrm{d} \tau+L(\boldsymbol{X}(\tau), \tau) \mathrm{d} \boldsymbol{Y},
$$

where the vector $\boldsymbol{X}(\tau), \boldsymbol{f}$ and the matix $L$ are given by

$$
\begin{aligned}
X & =\left(\begin{array}{l}
X_{1} \\
X_{2}
\end{array}\right), \quad f=\left(\begin{array}{c}
X_{2} \\
-D_{1} X_{1}-C_{1} X_{2}
\end{array}\right), \\
L & =\left[\begin{array}{cc}
0 & 0 \\
\frac{R V_{j i} H_{j i}}{\operatorname{Pr} C_{j i} F_{j i}} X_{1} & 0
\end{array}\right]
\end{aligned}
$$

with $X_{1}(\tau)=B_{i}(\tau)$ and $X_{2}(\tau)=\dot{X}_{1}(\tau)$. The reason for choosing a white noise process is two-fold. First, this is one of the most commonly used stochastic processes which is amenable to a fairly simple and straighforward mathematical analysis. Hence we use it as an approximation to a number of random physical phenomena. Secondly, the use of white noise models simulates well the behaviour of the actual stochastic process if one deals with a small portion of the frequency spectrum in the analysis (Soong [24]). The term "white" is introduced in connection with the white light, which has the property that its power spectral density is flat over the visible portion of the electromagnetic spectrum. However it is customary to define noise in a stronger sense so that its power spectral density is constant for all frequencies. It is important to mention here that in the deterministic case, the linear stability analysis for Bénard convection fails (see Venezian [25], Gresho and Sani [26], Rosenblat and Herbert [27], Rosenblat and Tanaka [28]) in the limit of low frequency of the wall temperature on gravity modulation. In particular, Venezian [25] pointed out that nonlinear effects may become important in the low frequency limit when the temperatures of the plates (within which convection occurs) are oscillated out of phase or when only plate temperature is modulated. In the present study with random forcing of the white noise type, in our opinion the detailed analysis for the low frequency limit is not relevant.

The vector $\boldsymbol{Y}(\tau), \tau \geqq 0$ defined in (12) represents a two-dimensional Brownian motion process (or a Wiener process) with zero mean. If $p(X(\tau), \tau)$ is an arbitrary function of $\boldsymbol{X}(\tau)$ and $\tau$, the following moment 
equation can be derived from (12) (Soong [24]):

$$
\begin{aligned}
\frac{\mathrm{d}}{\mathrm{d} \tau} E\{p(\boldsymbol{X}(\tau), \tau)\} & =\sum_{j=1}^{2} E\left\{f_{j} \frac{\partial p}{\partial \varkappa_{j}}\right\} \\
+ & \sum_{i, j=1}^{2} E\left\{\left(L D L^{\mathrm{T}}\right)_{j i} \frac{\partial^{2} p}{\partial \varkappa_{i} \partial \varkappa_{j}}\right\}+E\left\{\frac{\partial p}{\partial \tau}\right\},
\end{aligned}
$$

where $E$ stands for mathematical expectation and $D$ stands for the $2 \times 2$ matrix with elements $D_{i j}$ satisfying

$$
E\left\{\Delta Y_{i}(\tau) \Delta Y_{j}(\tau)\right\}=2 D_{i j} \Delta \tau, \quad i, j=1,2 .
$$

Here $\Delta Y_{i}(\tau)\left(=Y_{i}(\tau+\Delta \tau)-Y_{i}(\tau)\right)$ is an increment in $Y_{i}(\tau)$ during the time interval $\Delta \tau$.

Introducing the moment

$$
m_{j k}(\tau)=E\left\{X_{1}^{j}(\tau) X_{2}^{k}(\tau)\right\},
$$

we find from (14) the first-order moment equations as

$$
\begin{aligned}
& \dot{m}_{10}(\tau)=m_{01}(\tau), \\
& \dot{m}_{01}(\tau)=-D_{1} m_{10}(\tau)-C_{1} m_{01}(\tau) .
\end{aligned}
$$

Similarly the second-order moment equations are

$$
\begin{aligned}
\dot{m}_{20}(\tau) & =2 m_{11}(\tau), \\
\dot{m}_{11}(\tau) & =-D_{1} m_{20}(\tau)-C_{1} m_{11}(\tau)+m_{02}(\tau), \\
\dot{m}_{02}(\tau) & =2\left[D_{11}\left(\frac{R V_{j i} H_{j i}}{\operatorname{Pr} C_{j i} F_{j i}}\right)^{2} m_{20}(\tau)\right. \\
& \left.-D_{1} m_{11}(\tau)-C_{1} m_{02}(\tau)\right],
\end{aligned}
$$

where $D_{11}$ is the only nonzero element in $D_{i j}$ for a white noise process. As these equations are linear, the solutions are assumed in the form

$$
m_{10}(\tau)=\sum_{j=1}^{2} \alpha_{j} e^{\lambda_{j} \tau}, \quad m_{01}(\tau)=\sum_{j=1}^{2} \beta_{j} e^{\lambda_{j} \tau}
$$

For asymptotic stability in the mean, the real parts of $\lambda_{j}$ must be negative. Using (19) and (17) we find that for nontrivial solutions $\lambda_{j}$ must be satisfy

$$
\lambda^{2}+C_{1} \lambda+D_{1}=0 .
$$

Thus the requirements for asymptotic stability in the mean are

$$
C_{1}>0 \text { and } D_{1}>0 .
$$

Proceeding in the same way, the criterion for asymptotic mean squares stability can be obtained. The characteristic equations associated with the second order moment equations (18) reduced to

$$
\begin{aligned}
\lambda^{3}+3 C_{1} \lambda^{2}+ & 2\left(C_{1}^{2}+2 D_{1}\right) \lambda \\
& +4\left(C_{1} D_{1}-\frac{D_{11} R^{2} V_{j i}^{2} H_{j i}^{2}}{\operatorname{Pr}^{2} C_{j i}^{2} F_{j i}^{2}}\right)=0 .
\end{aligned}
$$

Using the Routh-Hurwitz criterion, we find that the second-order moments are stable if all the coefficients in (21) are positive and the inequality

$$
6 C_{1}\left(C_{1}^{2}+2 D_{1}\right)>4\left(C_{1} D_{1}-\frac{D_{11} R^{2} V_{j i}^{2} H_{j i}^{2}}{\operatorname{Pr}^{2} C_{j i}^{2} F_{j i}^{2}}\right)
$$

holds. Using (11), we obtain the condition for stationary instability as:

$R^{2} D_{11} V_{j i}^{2} H_{j i}^{2}+\left[R V_{j i} H_{j i}+M H_{j i} U_{j i}-D_{j i} G_{j i}\right] L_{j i}<0$,

where $L_{j i}=D_{j i} F_{j i} P r+C_{j i} G_{j i}$. Assuming positive $R$, we have from (23)

$$
\begin{aligned}
R= & \left(2 D_{11} V_{j i}^{2} H_{j i}^{2}\right)^{-1}\left[-L_{j i} V_{j i} H_{j i}+\left\{V_{j i}^{2} H_{j i}^{2} L_{j i}^{2}\right.\right. \\
& \left.\left.+4 D_{11} L_{j i} V_{j i}^{2} H_{j i}^{2}\left(D_{j i} G_{j i}-M U_{j i} H_{j i}\right)\right\}^{1 / 2}\right] .
\end{aligned}
$$

Since positive $R$ values will be relevant in our case, the negative sign in front of the root is dropped. Now it can be shown that $\partial R / \partial D_{11}$ is always negative, which implies that as $D_{11}$ increases $R$ decreases, this means that the random parameters exerts a destabilizing influence on the system. As $D_{11} \rightarrow 0$, it follows from (24) on binomial expansion in powers of $D_{11}$ that

$$
R=\frac{1}{2 V_{j i}^{2} H_{j i}^{2}}\left[2 V_{j i} H_{j i}\left(D_{j i} G_{j i}-M U_{j i} H_{j i}\right)\right]
$$

which reduces to the form

$$
V_{j i} H_{j i} R+M U_{j i} H_{j i}=D_{j i} G_{j i}
$$

and coincides with the expression obtained by Finlayson [23] for the deterministic case. Since the qualitative results will not change on considering higher approximations for the functions $W$ and $\Theta$ and the parameters $R, M$ etc. come out fairly accurately (Chandrasekhar [22]), so by considering $i, j=1,1$ and omitting the suffix we can write

$M=-\frac{D_{11} V^{2} H^{2}}{U(\operatorname{Pr} D F+C G)} \cdot R^{2}-\frac{V}{U} R+\frac{D G}{U H}$,

which represents a parabola in the $(R-M)$ plane of the form

$$
y=a_{1} \varkappa^{2}+b_{1} \varkappa+c_{1} .
$$


It should be emphasized here that by different choices of the trial functions for $\Theta_{1}(z)$ and $W_{1}(z)$ one may obtain different values of $a_{1}, b_{1}$ and $c_{1}$.

It may be interesting to draw the phase diagram of (10) for fixed values of $R, P r$ and the random parameter $D_{11}$. Two sets of values of $M\left(M_{1}\right.$ and $M_{2}$ (say)) are chosen such that $M_{1}<M^{\mathrm{c}}$ and $M_{2}>M^{\mathrm{c}}$ is the critical value of $M$ for the above fixed parameters.

For $i=j=1,(10)$ can be written in the form

$$
\ddot{x}+a \dot{x}+(b-c) x=0,
$$

where $a, b$, and $c$ will be constant for fixed $R, P r, D_{11}$, and $M$. Now, (29) can be recast in an equivalent system of equations as

$$
\dot{X}=Y, \quad \dot{Y}=(c-b) X-a Y,
$$

and the characteristic equation will have the root

$$
K_{1,2}=\frac{-a \pm \sqrt{a^{2}+4(c-b)}}{2} .
$$

It is clear from (10) and (11) that the values of $a$ and $c$ are always positive and are independent of $M$, but $b$ will change for different values of $M$. It can also be shown from (11) that $\left[a^{2}-4(b-c)\right]$ is always positive, irrespective of the values of $R$ and $M$.

Depending on $M$ the following cases are to be considered:

Case 1. If $(c-b)<0$, then both $K_{1}$ and $K_{2}$ of (31) are real and negative. The rest point is an asymptotically stable nodal point. All solutions are damped and nonoscillating. Figure 1 depicts the arrangement of trajectories about a rest point. The arrows indicate the direction of motion along the trajectories as $t$ increases. Here, $M=M_{1}$ and $M_{1}$ may take any value except those values for which $(c-b)=0$.

Case 2. If $(c-b)=0$, then $K_{1}=0$ and $K_{2}<0$. The rest point is stable, but not in the asymptotic sense. Figure 2 shows the trajectories for this case. As $t \rightarrow \infty$ the points on every trajectory approach the rest point $X=C_{1} \alpha_{1}$ and $Y=C_{1} \alpha_{1}$ located on the straight line $\alpha_{1} Y=\alpha_{2} X$, where $C_{1}$ is an arbitrary constant and $\alpha_{1}$ and $\alpha_{2}$ are constants that can be determined from (30) (Elsgolts [29]). Here $M=M_{1} \cong M^{\mathrm{c}}$, for our first approximation $M^{\mathfrak{c}}=57$ (approx.) and $M_{1}=56.7849$ (approx.).

Case 3. If $(c-b)>0$, then $K_{1}>0$ and $K_{2}<0$ are two real roots. The rest point in this case is unstable. Fig-

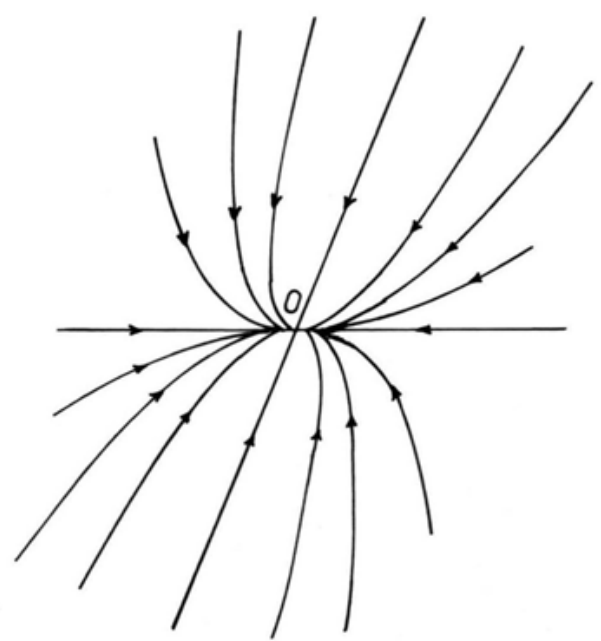

Fig. 1. Arrangement of trajectories for $(c-b)<0$, here $M=M_{1}$ which can take any value except $(c-b)=0$.

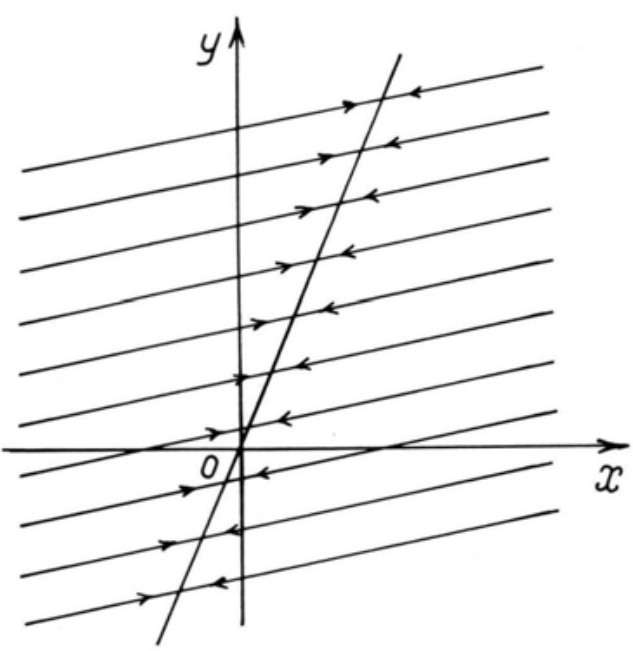

Fig. 2. Arrangement of trajectories for $(c-b)=0$, here $M=$ $M_{1} \cong M^{\mathrm{c}}$.

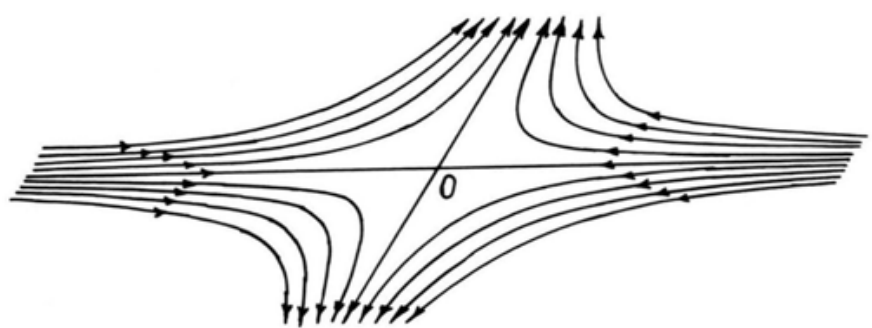

Fig. 3. Arrangement of trajectories for $(c-b)>0$, here $M=$ $M_{2} \cong M^{\mathrm{c}}$. 
ure 3 depicts the trajectories of the points. A rest point of this type is called a saddle point. For this case $M=M_{2}>M^{\mathrm{c}}$, where $M_{2}$ may take any value.

\section{Discussion}

Destabilizing influences of $D_{11}$ deserve some comments and a physical explanation for the destabilizing effects of radom vibrations on convection may be offered as follows. Ignoring diffusive effects, a random gravitational forcing superimposed on the steady value, increases the mean squares value of gravity and so makes disturbances to the system grow more rapidly. If, however, the fluctuating part has a carefully chosen frequency it may be possible to stabilize the system in a way similar to the inverted pendulum. Thus, fixed (and hence deterministic) frequency oscillations may stabilize, but random ones will destabilize the system. It is well known that deterministic oscillations may either stabilize or destabilize a system depending on the amplitude and frequency of the imposed oscilla-

[1] H. Bénard, Rev. Gen. Sci. Pure Appl. 11, 1261, 1309 (1900).

[2] Lord Rayleigh, Phil. Mag. 32, 529 (1916).

[3] H. Bénard, Proc. 3rd Int. Congr. Appl. Mech. 1, 120 (1930).

[4] M. J. Block, Nature London 178, 650 (1956).

[5] J. R. Pearson, J. Fluid Mech. 4, 489 (1958).

[6] D. A. Nield, J. Fluid Mech. 19, 341 (1964)

[7] L. E. Scriven and C. V. Sternling, J. Fluid Mech. 19, 321 (1964).

[8] S. H. Davis, J. Fluid Mech. 39, 347 (1969).

[9] S. H. Davis and M. G. Homsy, J. Fluid Mech. 98, 527 (1980).

[10] J. L. Castillo and M. G. Velarde, J. Fluid Mech. 125, 463 (1982).

[11] J. W. Scanlon and L. A. Segel, J. Fluid Mech. 30, 149 (1967).

[12] J. R. Kraska and R. D. Sani, Int. J. Heat and Mass Trans. 22, 535 (1979).

[13] S. Rosenblat et al., J. Fluid Mech. 120, 91, 123 (1982).

[14] A. Cloot and G. Lebon, J. Fluid Mech. 145, 447 (1984).

[15] G. Ahlers, M. C. Cross, P. C. Hohenberg, and S. Safran, J. Fluid Mech. 10, 297 (1981)

[16] L. A. Segel, J. Fluid Mech. 38, 203 (1969).

[17] A. C. Newell and J. A. Whitehead, J. Fluid Mech. 38, 279 (1969). tions (Yih and $\mathrm{Li}[30]$ ). In their study, they found that a time-periodic temperature modulation destabilizes the system over a wide range of modulation frequencies while at low modulation frequency it stabilizes the system. We may expect that in a thermal stability analysis based on a multichromatic disturbance (i.e. a disturbance averaged over all frequencies) modulation may have a net destabilizing influence since the stabilizing influence of modulation over a small part of the frequency spectrum may be overwhelmed by the destabilizing influence over a much wider range of frequencies. This prediction for deterministic modulation parallels the result predicted in the present investigation, as the random vibrations of the white noise type may be regarded as a modulation averaged over all frequencies.

\section{Acknowledgement}

The author expresses his sincere thanks to Professor A. S. Gupta and Professor B. B. Rao for their help in clarifying certain aspects of this work.

[18] C. W. Meyer, G. Ahlers, and D. S. Cannell, Phys. Rev. Lett. 59, 1577 (1987).

[19] V. Zaitsev and M. Shliomis, Sov. Phys., J. Exp. Theor. Phys. 32, 866 (1971).

[20] B. Jhaveri and G. M. Homsy, J. Fluid Mech. 98, 329 (1980).

[21] S. Ostrach, Ann. Rev. Fluid Mech. 14, 313, Ann. Rev. Inc., Palo Alto, California, USA 1982.

[22] S. Chandrasekhar, Hydrodynamic and Hydromagnetic Stability, Oxford University Press, 1961.

[23] B. A. Finlayson, The Method of Weighted Residuals and Variational Principles, Academic Press, London 1972.

[24] T. T. Soong, Random Differential Equations in Science and Engineering, Academic Press, London 1973.

[25] G. Venezian, J. Fluid Mech. 35, 243 (1969).

[26] P. M. Gresho and R. L. Sani, J. Fluid Mech. 40, 783 (1970).

[27] S. Rosenblat and D. M. Herbert, J. Fluid Mech. 43, 385 (1970).

[28] S. Rosenblat and G. A. Tanaka, Phys. Fluids 14, 1319 (1971).

[29] L. Elsgolts, Differential Equations and Calculus of Variations, Mir Publishers, Moscow 1973.

[30] C. S. Yih and C. H. Li, J. Fluid Mech. 54, 143 (1972). 